\title{
Ex vivo Evaluation of Caries Infiltration after Different Application Times in Primary Molars
}

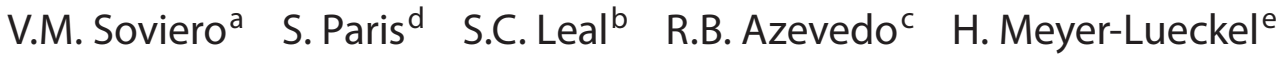 \\ a Department of Preventive and Community Dentistry, Universidade do Estado do Rio de Janeiro, Rio de Janeiro, \\ and ${ }^{b}$ Department of Dentistry, Faculty of Health Science, and ${ }^{c}$ Department of Genetics and Morphology,

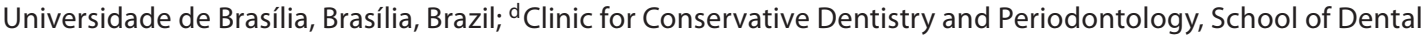 \\ Medicine, Christian-Albrechts-Universität zu Kiel, Kiel, and e Department of Operative Dentistry, Periodontology \\ and Preventive Dentistry, Rheinisch-Westfälische Technische Hochschule Aachen, Aachen, Germany
}

\section{Key Words}

Bitewing $\cdot$ Caries infiltration $\cdot$ Dental caries $\cdot$ Polarized light microscopy $\cdot$ Scanning electron microscopy

\begin{abstract}
Low viscosity resins (infiltrants) have been shown to penetrate the lesion body of natural caries lesions almost completely in vitro. However, penetration depths (PD) have not been evaluated in vivo. Therefore, the aim of the present study was to evaluate the penetration of an infiltrant into proximal caries lesions in primary molars after different application times using an ex vivo model. 59 proximal lesions from 34 children were randomly allocated to one of the application times and were infiltrated under clinical conditions for 1,3 , or 5 min. After extraction or exfoliation $(n=48)$, teeth were sectioned perpendicular to their surfaces and lesion depths (LD) as well as lesion areas (LA) were evaluated using polarized light microscopy. PD and penetration areas (PA) were measured on scanning electron microscopic images. Percentage penetration depth (PPD) and percentage penetration area (PPA) were calculated. The mean $( \pm S D) L D$ and LA were $596 \pm 203 \mu \mathrm{m}$ and $4.03 \pm 2.75 \times 10^{5} \mu \mathrm{m}^{2}$, respectively. PPD ranged from 70 to $80 \%$ and PPA from 54 to $60 \%$. Longer application times did not result in significantly deep-
\end{abstract}

er or more complete penetration ( $p>0.05$; ANOVA). In conclusion, proximal caries lesions in primary molars can be infiltrated in vivo to a similar extent as observed previously in vitro. Moreover, 1-min application of the infiltrant led to PD and homogeneity similar to those observed with longer application times up to 5 min.

Copyright $\odot 2012$ S. Karger AG, Basel

The prevalence of children with initial proximal caries in primary molars varies from 33 to $75 \%$ in low and high caries prevalence groups, respectively [Anderson et al., 2005; Nobre Dos Santos et al., 2005; Lillehagen et al., 2007]. In the absence of cavitation a noninvasive approach based on plaque control, fluoridation, and dietary counseling has been recommended [Kidd and Fejerskov, 2004]. However, if good compliance of the patient cannot be achieved, proximal lesions often progress and require restorative treatment [Mejàre, 2000; Vanderas et al., 2006].

Fissure sealants are effective in arresting noncavitated caries lesions in occlusal surfaces [Griffin et al., 2008; Bakhshandeh et al., 2011; Borges et al., 2011]. Promising results were observed after sealing proximal caries with fissure sealants, adhesives, or polyurethane patches [Martignon et al., 2006, 2010; Alkilzy et al., 2011]. More-

\section{KARGER}

Fax +4161306 1234

E-Mail karger@karger.ch

www.karger.com (c) 2012 S. Karger AG, Basel

0008-6568/13/0472-0110\$38.00/0

Accessible online at:

www.karger.com/cre
Prof. Dr. Vera Mendes Soviero

Department of Preventive and Community Dentistry, Pediatric Dental Clinic Faculty of Dentistry, Universidade do Estado do Rio de Janeiro

Boulevard Vinte de Oito de Setembro, $157-2^{\circ}$ andar - Clínica de Odontopediatria 20551-030 Rio de Janeiro, RJ (Brazil), E-Mail verasoviero@gmail.com 
over, for clinical sealing of proximal lesions, two visits are required: the first to separate teeth with orthodontic elastic rings and the second for sealant application [Martignon et al., 2010].

In contrast to sealing where a protective coat is created on the enamel surface, infiltration aims to penetrate a low viscosity resin into the lesion body [Meyer-Lueckel and Paris, 2008a]. It has been shown in vitro [Meyer-Lueckel and Paris, 2008b] and in situ [Paris and Meyer-Lueckel, 2010] that a covering resin coat on the enamel surface is not essential to inhibit caries progression. However, only shallow penetration of adhesives was observed in natural caries lesions in vitro [Meyer-Lueckel and Paris, 2008a]. Furthermore, the efficacy of caries infiltration in reducing caries progression has been reported in clinical studies [Ekstrand et al., 2010; Paris et al., 2010; Martignon et al., 2012; Meyer-Lueckel et al., 2012].

In laboratory studies, a 5-min application time of the infiltrant resulted in the best penetration results [MeyerLueckel and Paris, 2008a]. In vitro studies [Meyer-Lueckel et al., 2011] showed that 3-min application was sufficient to infiltrate the lesion body of natural caries lesions in permanent teeth almost completely. In primary molars, although a tendency for deeper penetration of the infiltrant after longer application times was observed, enamel lesions were nearly completely infiltrated already after $1 \mathrm{~min}$ in vitro. Thirty-second application resulted in significantly shallower penetration depths (PD) of the infiltrant [Paris et al., 2011b].

As in vitro studies are not able to mimic the clinical situation completely, the current study aimed to evaluate the $\mathrm{PD}$ of an infiltrant applied in vivo on proximal lesions in primary molars after different application times. This is the first study to evaluate resin infiltration depths using an ex vivo model. The overall hypothesis of the present study was that with longer application times significantly deeper and more complete penetration of the infiltrant into natural proximal caries lesions in primary molars would be observed.

\section{Materials and Methods}

This study was designed as an ex vivo study with a randomized design and blind assessment. Ethical approval was given by the Ethics Committee of the University of the State of Rio de Janeiro, Brazil (2225-CEP/HUPE).

Sample

123 children (aged 9-12 years) were screened from January to December 2010 at the pediatric dental clinic at the University of the State of Rio de Janeiro and at the University of Brasília, both situated in Brazil. They were referred to bitewing examination because they had one or more active caries lesions as detected visually. Based on bitewing radiographs, children who presented at least one initial proximal caries lesion in a primary molar that was expected to exfoliate within the next 12 months were considered eligible for the study. Proximal lesions (fig. 1a, d) were visually assessed by two calibrated investigators with the naked eye using a light box. Caries lesions were scored as radiolucency into the outer half of enamel (E1), the inner half of enamel (E2), the outer third of dentin (D1), the middle third of dentin (D2), and the inner third of dentin (D3). In case of disagreement a consensus was achieved. Teeth having E2 and D1 lesions and being in contact with the respective adjacent tooth were included in the study. Cavitated (into dentin) caries lesions as assessed by visual-tactile examination were excluded. At maximum one proximal caries lesion per tooth was selected. The aims of the study were explained to parents before they gave written informed consent.

The sample size was calculated on the basis of a pilot evaluation of the current data after having included 15 lesions of which 14 could be assessed in vitro. The sample size calculation based on the mean percentage penetration depths (PPD) of these 14 lesions ( $\mathrm{n}=4-6$ per group) revealed that 33,14 , and 94 specimens were needed to obtain significant differences between 1/3-min, 1/5-min, and 3/5-min application times, respectively $(\alpha=0.05$; $1-\beta=0.8 ; \chi^{2}$ test). Thus, the goal was to assess at least 14 caries lesions for each application time, which resulted in $14 \times 3$ groups $=42$ caries lesions. We assumed a dropout and technical failure rate of $30 \%$, resulting in a sample of 60 caries lesions.

59 proximal caries lesions from 34 children (mean age $9.8 \pm$ 0.8 years) were treated. Three lesions were classified as E1, 36 as E2, and 20 as D1. Three E1 lesions were included in the sample because they were just between E1 and E2 and the patients presented active caries in other tooth surfaces.

\section{Intervention}

Lesions were allocated to one of three application times $(1,3$, or $5 \mathrm{~min}$ ) using computer-generated randomly permuted blocks of size 9 (sealed in envelopes) by an assistant. When more than one lesion was selected in the same child, lesions were considered following the sequence of examination from tooth 55 to tooth 65 and from tooth 75 to tooth 85 . Eighteen lesions were treated for $1 \mathrm{~min}, 21$ for $3 \mathrm{~min}$, and 20 for $5 \mathrm{~min}$.

Proximal caries lesions were treated with the infiltration technique using Icon (DMG, Hamburg, Germany) by one trained investigator according to the manufacturer's instructions except for variation of the application time $(1,3$, or $5 \mathrm{~min})$ and single infiltrant application (manufacturer recommends second application). The clinical procedure was as follows: (1) topical anesthesia on the gingival papilla followed by local anesthesia on the gingival papilla (only if necessary in order to apply the clamp on the first permanent molar sufficiently); (2) application of a rubber dam; (3) insertion of the plastic wedge for immediate tooth separation; (4) surface etching for $120 \mathrm{~s}$ with $15 \% \mathrm{HCl}$ gel using the foil applicator included in the treatment kit, which protects the adjacent surface from the acid; (5) rinsing with a water spray for $30 \mathrm{~s}$; (6) drying with compressed air for $30 \mathrm{~s}$; (7) desiccation of the lesion with $100 \%$ ethanol for $30 \mathrm{~s}$ and subsequent air blowing for $30 \mathrm{~s}$; (8) application of the infiltrant for 1, 3, or 5 min using the foil applicator; (9) removal of excess material from the lesion surface 

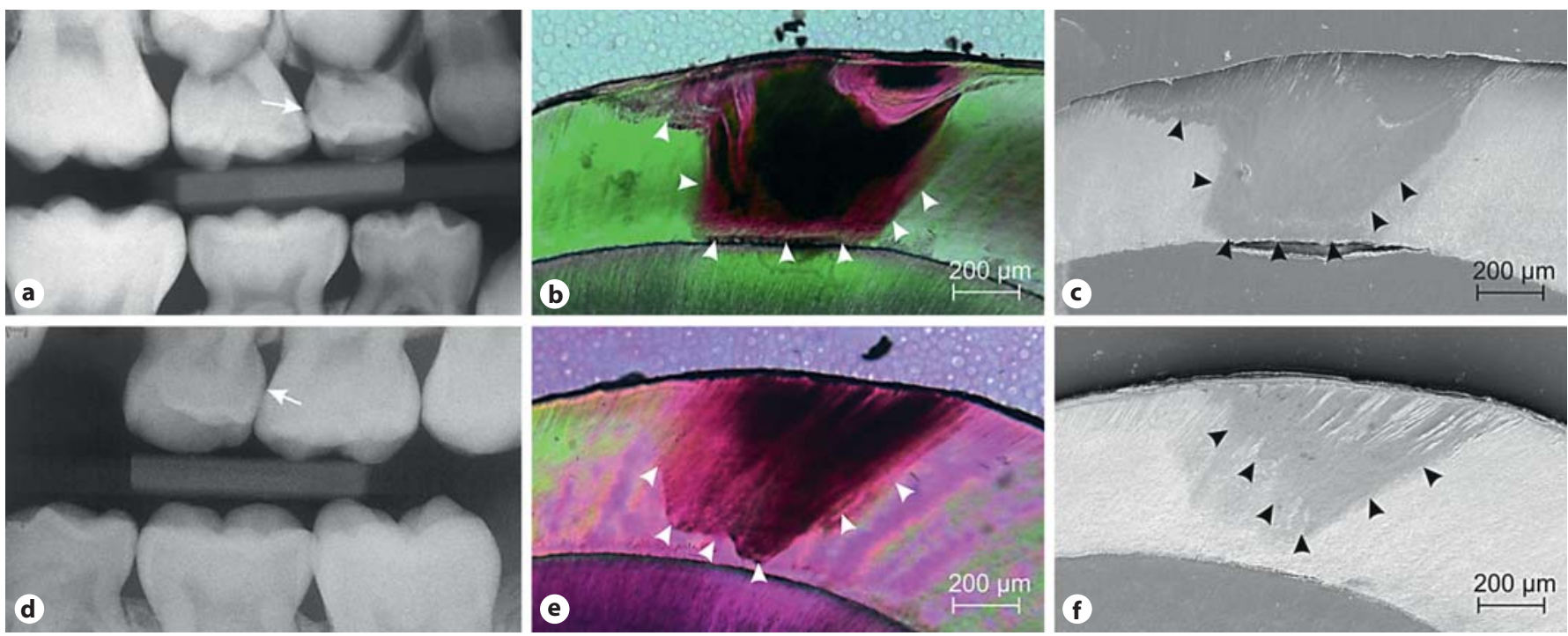

Fig. 1. Arrows indicate a radiological D1 (outer third of dentin) caries lesion on the distal surface of tooth 54 (a) and an E2 (inner enamel) caries lesion on the distal surface of tooth 64 (d). Arrowheads indicate the limits of the LA and PA of both caries lesions in polarized microscopic images (b, e) and SEM images (c, f), respectively. Almost complete (c) as well as partial (f) infiltration could be observed after 5- and 1-min application of the infiltrant, respectively.

by air blowing and dental floss; (10) light curing of the infiltrant with blue light for $60 \mathrm{~s}\left(350-500 \mathrm{~mW} / \mathrm{cm}^{2}\right.$, UltraLux; Dabiatlante, Ribeirão Preto, Brazil).

Teeth were either extracted immediately after treatment (if roots were almost completely reabsorbed or for orthodontic reasons) or followed until they could be extracted or were naturally exfoliated. Teeth were stored in $0.1 \%$ thymol solution.

In the follow-up visits, children and parents were questioned about possible adverse effects such as tooth pain or any discomfort in soft tissues around the infiltrated teeth.

\section{Analysis of the PD of the Infiltration}

Proximal caries lesions were scored by direct visual examination using ICDAS criteria [Pitts, 2004] by one trained examiner. For further analysis teeth were embedded in acrylic resin, and 130 - to $150-\mu \mathrm{m}$ transversal thin sections were prepared (BandSaw-300cl; Exakt-Apparatebau, Norderstedt, Germany). Tooth sections were polished up to a $100-\mu \mathrm{m}$ thickness (Mikroschleifsystem-400cs, abrasive paper 1,200, 2,400, and 4,000; Exakt-Apparatebau) and examined with polarized light microscopy (Microphot-FXA; Nikon, Tokyo, Japan). Sections showing the deepest part of each lesion were selected for measurement of the maximum lesion depth (LD) and lesion area (LA) (AxioVision-3.1; Carl Zeiss, Oberkochen, Germany). LD was measured along the enamel prisms from the tooth surface up to the deepest point of the lesion in the enamel (fig. 1b, e). Subsequently, the same sections were etched with $10 \% \mathrm{HCl}$ solution for $30 \mathrm{~s}$, washed with deionized water, dried at room temperature for $24 \mathrm{~h}$, and analyzed using scanning electron microscopy (SEM) in backscattered electron mode (Philips-XL30; Eindhoven, The Netherlands) [Paris et al., 2009]. On the SEM images the PD of the infiltrant (along the enamel prisms at the deepest point of the lesion) and the infil- trated area (PA) were measured (AxioVision-3.1) (fig. 1c, f). Measurements of LD and PD were done blindly regarding the application time.

\section{Statistical Analyses}

The PPD $(\mathrm{PPD}=\mathrm{PD} / \mathrm{LD} \times 100)$ and the percentage penetration area $(\mathrm{PPA}=\mathrm{PA} / \mathrm{LA} \times 100)$ were calculated. $\mathrm{PD}, \mathrm{PPD}$, and PPA were compared between the applications times. Subgroup analysis was done for lesions with LD $\geq 400 \mu \mathrm{m}$. Normal distribution was checked (Shapiro-Wilk test). Differences in LD, PD, PPD, and PPA were analyzed using one-way ANOVA and tests (SPSS-19.0; Chicago, Ill., USA). The level of significance was set at 5\%. Intraexaminer reliability for measurements of LD, LA, PD, and PA was assessed by repeated analysis of 20 lesions using the intraclass correlation coefficient (ICC).

\section{Results}

At the follow-up visits none of the children reported pain or any other discomfort related to the infiltrated teeth. Of the 59 treated teeth, 22 were extracted immediately after infiltration, 14 were extracted/exfoliated in the first 5 months, and 18 were extracted 6-12 months after infiltration. Five teeth could not be collected because parents did not keep them after exfoliation. Six teeth broke during preparation. The final sample consisted of 48 teeth (table 1). 
Table 1. Data of the 59 proximal lesions (by application time), including the final sample $(\mathrm{n}=48)$ and the lost teeth $(\mathrm{n}=11)$

\begin{tabular}{|c|c|c|c|c|c|c|}
\hline & \multicolumn{3}{|c|}{ Sample $(\mathrm{n}=59)$} & \multicolumn{2}{|l|}{ Lost } & \multirow{2}{*}{$\begin{array}{l}\text { Final } \\
\text { sample } \\
(\mathrm{n}=48)\end{array}$} \\
\hline & $1 \mathrm{~min}$ & $3 \min$ & $5 \mathrm{~min}$ & $\begin{array}{l}\text { broken } \\
(\mathrm{n}=6)\end{array}$ & $\begin{array}{l}\text { dropout } \\
(\mathrm{n}=5)\end{array}$ & \\
\hline \multicolumn{7}{|l|}{ Tooth } \\
\hline 1st molar & 10 & 5 & 8 & 3 & 2 & 18 \\
\hline 2nd molar & 8 & 16 & 12 & 3 & 3 & 30 \\
\hline \multicolumn{7}{|l|}{ Jaw } \\
\hline Upper & 9 & 11 & 10 & 3 & 1 & 26 \\
\hline Lower & 9 & 10 & 10 & 3 & 4 & 22 \\
\hline \multicolumn{7}{|l|}{ Surface } \\
\hline Mesial & 2 & 5 & 4 & 1 & 3 & 7 \\
\hline Distal & 16 & 16 & 16 & 5 & 2 & 41 \\
\hline \multicolumn{7}{|l|}{ X-ray score } \\
\hline E1 & 1 & 1 & 1 & 1 & 0 & 2 \\
\hline E2 & 12 & 14 & 10 & 3 & 3 & 30 \\
\hline D1 & 5 & 6 & 9 & 2 & 2 & 16 \\
\hline \multicolumn{7}{|c|}{ Time to extraction or exfoliation } \\
\hline Immediately & 5 & 9 & 8 & 2 & 0 & 20 \\
\hline$\leq 5$ months & 3 & 6 & 5 & 1 & NA & 13 \\
\hline$>5$ months & 10 & 6 & 7 & 3 & & 15 \\
\hline \multicolumn{7}{|l|}{ ICDAS score } \\
\hline 1 & 4 & 3 & 3 & 1 & NA & 9 \\
\hline 2 & 8 & 10 & 9 & 1 & & 26 \\
\hline 3 & 3 & 5 & 1 & 2 & & 7 \\
\hline 4 & 1 & 0 & 3 & 1 & & 3 \\
\hline 5 & 1 & 1 & 2 & 1 & & 3 \\
\hline
\end{tabular}

E1 = Radiolucency confined to the outer half of enamel; E2 = radiolucency in the inner half of the enamel; D1 = radiolucency in the outer third of dentin; NA = information not available.

a Direct visual examination after extraction/exfoliation.

The mean $( \pm S D)$ LD and LA were $596 \pm 203 \mu \mathrm{m}$ and $4.03 \pm 2.75 \times 10^{5} \mu \mathrm{m}^{2}$, respectively, for all lesions $(\mathrm{n}=$ 48). For lesions with $L D \geq 400 \mu \mathrm{m}(\mathrm{n}=39)$ the overall mean LD and LA were $665 \pm 153 \mu \mathrm{m}$ and $4.63 \pm 2.68$ $\times 10^{5} \mu \mathrm{m}^{2}$, respectively. No differences regarding LD or LA between the three groups differing in application times were observed ( $p>0.05$; ANOVA; table 2). Measurements showed a high degree of intraexaminer reproducibility (ICC 0.980-0.996).

The infiltrant penetrated rather deeply in most of the proximal caries lesions even after a 1-min application time (table 2). No significant differences between the groups were observed ( $p>0.05$; ANOVA; table 2). The mean PPD ranged from 70 to $80 \%$ and the mean PPA from 54 to $60 \%$ for all lesions. Similar results were observed for lesions with $\mathrm{LD} \geq 400 \mu \mathrm{m}$. Longer application times did not result in significantly deeper percentage

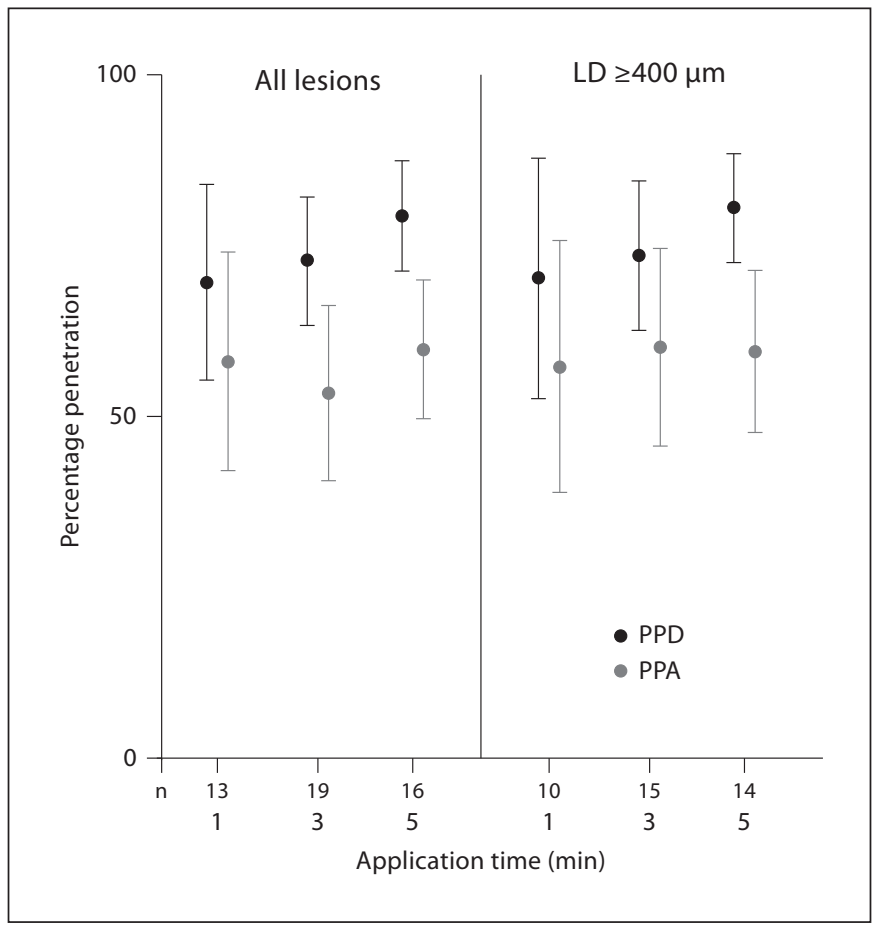

Fig. 2. Mean (error bars: 95\% confidence intervals) PPD and PPA of the infiltrant for the three application times for all lesions and for lesions with $\mathrm{LD} \geq 400 \mu \mathrm{m}$ ( $\mathrm{p}>0.05$; ANOVA). $\mathrm{n}=$ Number of teeth.

penetration ( $p>0.05$; fig. 2). PPD and PPA did not differ significantly between lesions confined radiographically into enamel (E1+E2) $(74 \pm 21$ and $57 \pm 26 \%$, respectively) compared with those extending into dentin (D1) (76 \pm 17 and $58 \pm 19 \%$, respectively) ( $p>0.05$; t test).

PPD and PPA were also compared between teeth extracted/exfoliated within shorter ( $\leq 5$ months) or longer ( $>5$ months) periods of time, but no significant differences were observed ( $p>0.05$; $t$ test). PPD and PPA were $76 \pm 18$ and $59 \pm 24 \%$, respectively, for teeth extracted/ exfoliated at $\leq 5$ months and $71 \pm 23$ and $52 \pm 25 \%$, respectively, for those that remained longer in the oral cavity.

\section{Discussion}

This was the first ex vivo study to investigate the penetration of an infiltrant into proximal caries lesions. Although in vitro studies have shown that natural proximal lesions can be almost completely infiltrated [MeyerLueckel and Paris, 2008a; Meyer-Lueckel et al., 2011; Par- 
Table 2. Means $( \pm S D$ ) of the maximum LD, LA, PD, and PA of the infiltrant with respect to the various application times for all lesions and for lesions with $\mathrm{LD} \geq 400 \mu \mathrm{m}$

\begin{tabular}{|c|c|c|c|c|c|c|c|c|c|c|}
\hline \multirow{2}{*}{$\begin{array}{l}\text { Application } \\
\text { time, min }\end{array}$} & \multicolumn{5}{|c|}{ All lesions } & \multicolumn{5}{|c|}{ Lesion with $\mathrm{LD} \geq 400 \mu \mathrm{m}$} \\
\hline & $\mathrm{n}$ & $\mathrm{LD}$ & $\mathrm{PD}$ & LA & $\mathrm{PA}$ & $\mathrm{n}$ & $\mathrm{LD}$ & $\mathrm{PD}$ & LA & PA \\
\hline 1 & 13 & $551 \pm 202$ & $387 \pm 206$ & $3.13 \pm 1.53$ & $1.72 \pm 1.06$ & 10 & $632 \pm 136$ & $451 \pm 190$ & $3.70 \pm 1.14$ & $2.07 \pm 0.95$ \\
\hline 3 & 19 & $577 \pm 200$ & $418 \pm 186$ & $3.91 \pm 2.17$ & $2.22 \pm 1.93$ & 15 & $655 \pm 142$ & $476 \pm 164$ & $4.51 \pm 2.02$ & $2.68 \pm 1.93$ \\
\hline
\end{tabular}

No significant differences were observed between application times ( $\mathrm{p}>0.05$; ANOVA). $\mathrm{n}=$ Sample size per group.

is et al., 2011a], it was necessary to confirm these findings in vivo, since in vitro studies cannot mimic the clinical situation completely. Nonetheless, the current study corroborated that proximal lesions in primary molars could be relatively homogeneously infiltrated even after a 1-min application time. Thus, the hypothesis that longer application times of 3 or 5 min result in significantly deeper and more complete penetration of the infiltrant has to be rejected at an adequate power at least for the comparison of 5- and 1-min application times.

The PD of the infiltrant has been usually evaluated with confocal laser scanning microscopy using staining techniques with tetramethylrhodamine isothiocyanate in vitro [Meyer-Lueckel and Paris, 2008a]. However, due to toxic effects it is not possible to use the same staining technique under in vivo conditions. Therefore, an alternative method employing SEM was used in the current study. This method has been used to measure the penetration of resins into artificial caries lesions previously [Davila et al., 1975; Gray and Shellis, 2002]. Moreover, the results obtained with the staining technique were highly correlated to those using the SEM, thus providing similar outcomes [Paris et al., 2009]. In the present study, the measurement of LD and infiltration depth showed high intraexaminer reproducibility.

In comparison to in vitro studies [Meyer-Lueckel and Paris, 2008a; Meyer-Lueckel et al., 2011] the PPD in the current study was slightly lower but still reached $70-80 \%$ on average. In general, around $60 \%$ of LA of all specimens were penetrated by the infiltrant, indicating moderate homogeneity. Under clinical conditions the presence of saliva proteins and remnants of biofilm inside the enamel porosities might inhibit complete infiltration. Although lower infiltration depths in comparison to in vitro results were somehow expected, good penetration of the infiltrant was observed.
In contrast to a previous in vitro study using permanent teeth [Meyer-Lueckel et al., 2011], longer application times (3-5 min) did not result in significantly deeper or more homogeneous infiltration compared with $1 \mathrm{~min}$. The results of the present study were similar to a previous in vitro study using primary teeth [Paris et al., 2011b]. For both, a tendency towards a deeper PD after longer application times was observed. In the present study a lower predictability of penetration was observed after a 1-min application time due to a wider range of PPD compared to 3- and 5-min application times. Nonetheless, no significant difference was found between the 1-min application time in comparison with 3 or $5 \mathrm{~min}$.

As the LD limits the penetration of the infiltrant, shallower lesions might be infiltrated completely more rapidly than deeper ones. To limit the influence of LD on the results a subanalysis was done with lesions deeper than $400 \mu \mathrm{m}$. Even among the deeper lesions, a relatively high percentage penetration of the infiltrant was observed independently of the application time. As the penetrability of the infiltrant also depends on the porosity volume of the lesion, active lesions are certainly more easily infiltrated. The thinner surface layer is more easily abraded during acid etching exposing almost completely the more porous structure in the subsurface. Children recruited for the current study had moderate to high caries experience, being considered at high risk for the development and progression of proximal caries in primary molars [Vanderas et al., 2006]. Therefore, we estimate that the proximal lesions were active and highly porous. This might have contributed to the observed relatively deep penetration after only a 1-min application.

In deeper noncavitated caries lesions already reaching the dentin, it might be speculated that the fluid from the dentin tubules could impede proper desiccation of the 
enamel and inhibit infiltration. However, in the current study infiltration depth was very similar in enamel $(\mathrm{E} 1+\mathrm{E} 2)$ and dentin lesions (D1), showing that radiographic dentin involvement was not a factor that resulted in hampered infiltration of the enamel. It is worth mentioning that proximal lesions restricted to enamel radiographically are most likely extended into dentin histologically [Espelid and Tveit, 1986]. However, as the clinical indication for caries infiltration is based on the LD according to X-ray, it is relevant to confirm that D1 lesions can be properly infiltrated.

After extraction/exfoliation, most of the proximal caries lesions (35/48) were noncavitated, confirming that the micro-invasive treatment had been properly indicated. Not surprisingly, some of these lesions were scored as ICDAS 1 probably because the resin penetration into the enamel subsurface may have partially masked the whitish appearance of the lesions [Kim et al., 2011]. On the other hand, 3 lesions were scored as ICDAS 5. As these 3 teeth scored as ICDAS 5 were not extracted immediately after infiltration, it is unclear if they were cavitated when they were treated or if they progressed to cavitation during the next months in situ. Additionally, 5 of 10 lesions scored as ICDAS 3 or 4 were not extracted right after infiltration. Thus, they might have shown micro-cavities when they were treated or had progressed during the in situ period of the study. Prior to infiltration based on radiographic and visualtactile examination, these teeth were considered as being noncavitated. However, it might be possible that some of the cavities were not detected clinically. It should be kept in mind that the proportion of cavitated lesions in primary molars can reach almost 30\% among D1 lesions [Pitts and Rimmer, 1992].
For ethical reasons no follow-up X-rays were done to evaluate lesion progression since the primary teeth were close to exfoliation. If lesion progression had occurred after infiltration, observation of lower PPD in the teeth that stayed longer in the oral cavity would have been expected. Nonetheless, PPD did not differ between teeth extracted/exfoliated after a shorter or longer period of time, suggesting that at least no relevant caries progression had occurred.

It can be concluded that initial noncavitated proximal caries lesions in primary molars can be infiltrated in vivo to a similar extent as observed in vitro. Moreover, 1-min application of the infiltrant seems to lead to PD and homogeneity similar to those with longer application times up to $5 \mathrm{~min}$.

\section{Acknowledgments}

The authors thank Regina Marquardt and Michael Stiebritz for their valuable contribution to this study. V.M.S., H.M.L., S.P., S.C.L. and R.B.A. designed the experiment. V.M.S. and S.C.L. performed the clinical procedures. V.M.S., H.M.L. and S.P. performed the laboratory and statistical analysis. V.M.S., H.M.L., S.P., S.C.L. and R.B.A. wrote the paper.

\section{Disclosure Statement}

The Charité - Universitätsmedizin Berlin holds US and European patents for an infiltration technique for dental caries lesions in which two of the authors (S.P. and H.M.L.) are appointed as inventors. These patents have been licensed by DMG. S.P. and H.M.L. receive royalties from this license as well as a research grant from DMG. V.M.S. received a research grant from DMG. S.C.L. and R.B.A. report no conflict of interest. The funder had no role in the study design, data collection and analysis, the decision to publish, or the preparation of the manuscript.

\section{References}

Alkilzy M, Berndt C, Splieth CH: Sealing proximal surfaces with polyurethane tape: threeyear evaluation. Clin Oral Investig 2011;15: 879-884.

-Anderson M, Stecksén-Blicks C, Stenlund H, Ranggård L, Tsilingaridis G, Mejàre I: Detection of approximal caries in 5-year-old Swedish children. Caries Res 2005;39:9299.

Bakhshandeh A, Qvist V, Ekstrand KR: Sealing occlusal caries lesions in adults referred for restorative treatment: 2-3 years of follow-up. Clin Oral Investig 2012;16:521-529.

\footnotetext{
Borges BC, De Souza Bezerra Araújo RF, Dantas Ekstrand KR, Bakhshandeh A, Martignon S: RF, De Araújo Lucena A, De Assunção Pinheiro IV: Efficacy of a non-drilling approach to manage non-cavitated dentin occlusal caries in primary molars: a 12 -month randomized controlled clinical trial. Int J Paediatr Dent 2012;22:44-51.

Davila JM, Buonocore MG, Greeley CB, Provenza DV: Adhesive penetration in human artificial and natural white spots. J Dent Res 1975;54:999-1008. Treatment of proximal superficial caries lesions on primary molar teeth with resin infiltration and fluoride varnish versus fluoride varnish only: efficacy after 1 year. Caries Res 2010;44:41-46.

Espelid I, Tveit AB: Clinical and radiographic assessment of approximal carious lesions. Acta Odontol Scand 1986;44:31-37.

-Gray GB, Shellis P: Infiltration of resin into white spot caries-like lesions of enamel: an in vitro study. Eur J Prosthodont Restor Dent 2002;10:27-32.
} 
-Griffin SO, Oong E, Kohn W, Vidakovic B, Gooch BF, Bader J, Clarkson J, Fontana MR, Meyer DM, Rozier RG, Weintraub JA, Zero DT: The effectiveness of sealants in managing caries lesions. J Dent Res 2008;87:169174.

-Kidd EA, Fejerskov O: What constitutes dental caries? Histopathology of carious enamel and dentin related to the action of cariogenic biofilms. J Dent Res 2004;83:C35-C38.

Kim S, Kim EY, Jeong TS, Kim JW: The evaluation of resin infiltration for masking labial enamel white spot lesions. Int J Paediatr Dent 2011;21:241-248.

Lillehagen M, Grindefjord M, Mejàre I: Detection of approximal caries by clinical and radiographic examination in 9-year-old Swedish children. Caries Res 2007;41:177-185.

-Martignon S, Ekstrand KR, Ellwood R: Efficacy of sealing proximal early active lesions: an 18 -month clinical study evaluated by conventional and subtraction radiography. Caries Res 2006;40:382-388.

-Martignon S, Tellez M, Santamaría RM, Gomez J, Ekstrand KR: Sealing distal proximal caries lesions in first primary molars: efficacy after 2.5 years. Caries Res 2010;44:562-570.

-Martignon S, Ekstrand KR, Gomez J, Lara JS, Cortes A: Infiltrating/sealing proximal caries lesions: a 3-year randomized clinical trial. J Dent Res 2012;91:288-292.
Mejàre I, Stenlund H: Caries rates for the mesial surface of the first permanent molar and the distal surface of the second primary molar from 6 to 12 years of age in Sweden. Caries Res 2000;34:454-461.

Meyer-Lueckel H, Chatzidakis A, Naumann M, Dorfer CE, Paris S: Influence of application time on penetration of an infiltrant into natural enamel caries. J Dent 2011;39:465-469.

Meyer-Lueckel H, Paris S: Improved resin infiltration of natural caries lesions. J Dent Res 2008a;87:1112-1116.

Meyer-Lueckel H, Paris S: Progression of artificial enamel caries lesions after infiltration with experimental light curing resins. Caries Res 2008b;42:117-124.

Meyer-Lueckel H, Bitter K, Paris S: Randomized controlled clinical trial on proximal caries infiltration: three-year follow-up. Caries Res 2012; 46:544-548.

Nobre Dos Santos M, Rodrigues LK, Peres RC, Yokoyama RT, Gavazzi JC, Gavião MB: Relationships between occlusal or free-smooth and approximal caries in mixed dentition. Acta Odontol Scand 2005;63:308-313.

- Paris S, Bitter K, Naumann M, Dörfer CE, Meyer-Lueckel H: Resin infiltration of proximal caries lesions differing in ICDAS codes. Eur J Oral Sci 2011a;119:182-186.
- Paris S, Bitter K, Renz H, Hopfenmuller W, Meyer-Lueckel H: Validation of two dual fluorescence techniques for confocal microscopic visualization of resin penetration into enamel caries lesions. Microsc Res Tech 2009;72: 489-494.

Paris S, Hopfenmuller W, Meyer-Lueckel H: Resin infiltration of caries lesions: an efficacy randomized trial. J Dent Res 2010;89:823826.

Paris S, Meyer-Lueckel H: Inhibition of caries progression by resin infiltration in situ. Caries Res 2010;44:47-54.

- Paris S, Soviero VM, Seddig S, Meyer-Lueckel H: Penetration depths of an infiltrant into proximal caries lesions in primary molars after different application times in vitro. Int J Paediatr Dent 2012;22:349-355.

Pitts N: 'ICDAS' - an international system for caries detection and assessment being developed to facilitate caries epidemiology, research and appropriate clinical management. Community Dent Health 2004;21:193-198.

- Pitts NB, Rimmer PA: An in vivo comparison of radiographic and directly assessed clinical caries status of posterior approximal surfaces in primary and permanent teeth. Caries Res 1992;26:146-152.

-Vanderas AP, Gizani S, Papagiannoulis L: Progression of proximal caries in children with different caries indices: a 4-year radiographic study. Eur Arch Paediatr Dent 2006; 7:148152. 